\title{
FREE BOUNDARY MINIMAL SURFACES AND OVERDETERMINED BOUNDARY VALUE PROBLEMS
}

\author{
NIKOLAI NADIRASHVILI AND ALEXEI V. PENSKOI
}

\begin{abstract}
In this paper we establish a connection between free boundary minimal surfaces in a ball in $\mathbb{R}^{3}$ and free boundary cones arising in a one-phase problem. We prove that a doubly connected minimal surface with free boundary in a ball is a catenoid.
\end{abstract}

\section{INTRODUCTION}

In this paper we investigate free boundary minimal surfaces in a three-dimensional ball, i.e. proper branched minimal immersions of a surface $M$

$$
u: M \longrightarrow \mathbb{B}^{3} \subset \mathbb{R}^{3}
$$

such that $u(M)$ meets the boundary sphere $\mathbb{S}^{2}=\partial \mathbb{B}^{3}$ orthogonally. It is a classical and developed subject, see e.g. the books [DHS10, DHT10a, DHT10b. A celebrated result due to J. C. C. Nitsche [Nit72] states that if $M$ is a disk then $u(M)$ is also a plane disk.

Actually, in the paper [Nit72] a stronger result is announced. Namely, that this statement holds for capillary surfaces and the angle between $u(M)$ and $\mathbb{S}^{2}$ is a constant. Details of the proof could be found in the paper [RS97].

Recently, the result due to J. C. C. Nitsche was generalized by A. Fraser and R. Schoen in the paper [FS15] to the case of a minimal disk satisfying the free boundary condition in a constant curvature ball of any dimension.

In this paper we prove that a free boundary doubly connected minimal surface in a three-dimensional euclidean ball is a piece of a catenoid.

Since (i) a minimal map could be parametrized by a conformal parameter, (ii) a double-connected domain is conformally equivalent to an annulus

$$
A=\{z|\rho<| z \mid<1\} \subset \mathbb{C},
$$

2010 Mathematics Subject Classification. 53A10, 35N25.

The work of the second author was partially supported by the Simons Foundation and by the Young Russian Mathematics award. 
and (iii) any ball could be transformed by a homotety to a unit ball, it is sufficient to consider a map from annulus (1) to the unit ball $\mathbb{B}_{1}^{3} \subset \mathbb{R}^{3}$ centered at the origin.

Theorem 1.1. Let $u: \bar{A} \longrightarrow \overline{\mathbb{B}}_{1}^{3} \subset \mathbb{R}^{3}$ be a proper branched minimal immersion such that $u \in C^{2}(A) \cap C^{1}(\bar{A}), u(\partial A) \subset \mathbb{S}^{2}=\partial \mathbb{B}_{1}^{3}$ and $u(A)$ meets $\mathbb{S}^{2}$ orthogonally. Then $u(A)$ is a part of a catenoid.

This result was conjectured by A. Fraser and M. Li in [FL14].

Recently A. Fraser and R. Schoen proved the existence of free boundary minimal surfaces in $\mathbb{B}^{3}$ which have genus 0 and $n$ boundary components, see the papers [FS16], see also [FPZ17].

Let us remark that A. Fraser and R. Schoen established in the papers [FS11, FS16] a remarkable connection between minimal surfaces with free boundaries in a ball and Riemannian metrics on surfaces with boundaries extremizing eigenvalues of the Steklov problem on these surfaces. Let us also remark that connections of spectral isoperimetry with minimal surfaces is known also for some other spectral problems, see the paper [NS15].

In this paper we establish by means of the Minkowski transformation a connection between free boundary minimal surfaces and the extremal domains on the sphere $\mathbb{S}^{2}$ for the Dirichlet problem. The last spectral problem is related to the one-phase free boundary problem for homogeneous functions defined in cones. By virtue of this connection we prove some new results for the one-phase free boundary problem.

The one-phase free boundary problem is a minimization of an integral

$$
J(v)=\int_{G \cap\{v>0\}}\left(|\nabla v|^{2}+1\right) d x \rightarrow \min ,
$$

where $v \geqslant 0$. It appears in some models related to the cavitational flow. If $v$ is a minimizer and $G^{+}=\{x \in G, v(x)>0\}$ then $v$ is a solution of the following overdetermined problem in $G$,

$$
\begin{cases}v \geqslant 0 & \text { in } G, \\ \Delta v=0 & \text { on } G^{+}, \\ |\nabla v|=C & \text { on } \partial G^{+} \cap G,\end{cases}
$$

where $C>O$ is a constant. H. W. Alt and L. A. Caffarelli proved in the paper AC81 that the question of regularity of the boundary in the one-phase free boundary problem could be reduced to the onephase problem in a cone. Let $K ß \mathbb{R}^{n}$ be an open ( $n$-dimensional) cone with a smooth lateral boundary. We are interested in the following overdetermined problem, 


$$
\begin{cases}\Delta v=0 & \text { in } K, \\ v=0 & \text { on } \partial K, \\ |\nabla v|=1 & \text { on } \partial K \backslash\{0\}\end{cases}
$$

where $v$ is a homogeneous degree 1 function. Let us emphasise that the unknowns here are both $v$ and $K$. One can define an energy for the solutions of system (3) related to $J$, see the papers AC81, CS05. A solution $v$ of (3) is called stable if it is stable with respect to this energy. L. A. Caffarlelli, D. Jerison and C. E. Kenig proved that in $\mathbb{R}^{3}$ the only stable solutions of (3) are linear functions, the correspondent cone $K$ is a half-space, see the paper CJK04. This result was extended to the dimension 4, see the paper [JS15]. On the other hand, D. De Silva and D. Jerison gave an example of a nontrivial energy minimizing solution in dimension 7, see the paper [DSJ09].

We show that result from the paper [CJK04] holds if we just assume that $K$ is a simply connected cone instead of the stability of the solution of system (3).

Theorem 1.2. Suppose that $v$ and $K \subset \mathbb{R}^{3}$ is a solution of system (3). Then

a) if $K \cap \mathbb{S}^{2}$ is diffeomorphic to a disk then $K$ is a half-space;

b) if $K \cap \mathbb{S}^{2}$ is diffeomorphic to an anulus then $\mathbb{R}^{3} \backslash K$ is a circular cone formed by lines with aperture $2 \arccos \tanh \alpha$, where $\alpha$ is a solution of the equation $\sqrt{\alpha} \tanh \alpha=1$.

The proof of Theorem 1.2 is based on the following involution on the space of homogeneous order 1 functions. Let $f$ be a homogeneous function of order 1 defined in a cone $K \beta \mathbb{R}^{3}$. Consider the surface $H_{f}=\nabla f(K)$ called the hérisson of $f$ (the notion was introduced in the paper [LLR88]). The following theorem which goes back to Minkowski, see [Bla21, § 78], holds:

Proposition 1.3. Let $f$ be a homogeneous function of degree 1 defined in a cone $K ß \mathbb{R}^{3}$. Let $N\left(H_{f}\right)$ be a Gauss map of the hérisson $H_{f}$. Then the map $N: H_{f} \longrightarrow \mathbb{S}^{2}$ is inverse at regular points of $H_{f}$ to $\nabla f: \mathbb{S}^{2} \longrightarrow H_{f}$. Moreover at a regular point $z \in H_{f}$ the sum of curvature radii of the surface $H_{f}$ is equal to the trace of the Hessian of $f$ at $N(z)$.

In particular, from the proposition follows that if $f$ is a harmonic function then $H_{f}$ is a minimal surface. It is interesting to notice that the minimality of $H_{f}$ follows also from the results of $\mathrm{H}$. Lewy, see the paper [Lew68]. 
Proposition 1.4. Let $v$ be a harmonic function defined in a domain $G ß \mathbb{R}^{3}$. Suppose that $\operatorname{rank}($ Hess $v)=2$ in $G$. Then the set $\nabla v(G)$ is a minimal surface in $\mathbb{R}^{3}$.

Proposition 1.4 also is a consequence of a deep theory of special Lagrangian manifolds, see the paper [HL82].

It is interesting to notice that by a remarkable observation of M. Traizet [Tra14] the entire solutions of the one-phase free boundary problem (2) on the plane are related to complete minimal surfaces in $\mathbb{R}^{3}$. Traizet constructed a Weierstrass-type map from entire solutions of (2) to immersed minimal surfaces in $\mathbb{R}^{3}$. Surfaces constructed by Traizet are symmetric with respect to a plane in $\mathbb{R}^{3}$ and hence they meet that plane orthogonally. It appears that for the simply connected $G$ there are only two entire solutions of (2) with the corresponding minimal surfaces a plane and a catenoid.

Notice that a restriction of homogeneous order 1 harmonic function $u$ on the sphere $\mathbb{S}^{2}$ is an eigenfunction of the Laplacian on $K \cap \mathbb{S}^{2}$ with the eigenvalue 2 . Thus we can set Theorem 1.2 with some generalizations in terms of overdetermined spectral problem. Let $\Omega$ be a bounded two dimensional simply connected Riemannian surface of a constant Gaussian curvature $\kappa$ and with a smooth boundary $\partial \Omega$. For the Laplace-Beltrami operator $\Delta$ on $\Omega$ suppose $v$ be a solution of the following overdetermined spectral problem:

$$
\begin{cases}\Delta v=\lambda v & \text { in } \Omega, \\ v=\alpha & \text { on } \partial \Omega, \\ |\nabla v|=\beta & \text { on } \partial \Omega,\end{cases}
$$

It is expected that nontrivial solutions of system (44) exist only in a disk. In the flat case and $\beta=0$ that conjecture is known as the Schiffer's conjecture. Its generalization (generalized Schiffer's conjecture) was widely discussed, see SSch01]. It has a dual integral-geometrical setting, [WCG95]. In the plane case $(\kappa=0)$ for $\beta=0$ the above conjecture is equivalent to a long standing Pompeiu conjecture, see details in a beautiful survey of L. Zalcman [Zal80]. On symmetric spaces the problem was discussed in the paper [BZ80]. The case of the unbounded domain $\Omega$ was discussed in the paper [BCN97]. For a flat unbounded $\Omega$ and $\lambda=\alpha=0$ a nontrivial example of a solution of (44) comes from the catenoid via the Traizet map. However, for bounded solutions $v$ the conjecture holds, see the paper [RRS17.

We prove the following

Theorem 1.5. Assume $\lambda=-2 \kappa$ and $v$ is a solution of system (4). Then $\Omega$ is a geodesic disk. 


\section{Proofs of the theorems}

Lemma 2.1. If the Gaussian curvature of a free-boundary mininal surface in a three-dimensional ball is constant on a connected component of a boundary, then this component of the boundary is an arc of a circle.

Proof. We can assume that the ball is of radius 1 . Let $N$ denote a unit normal field on the surface. Let us choose a point $p$ on the component of the boundary. Since the surface meets the boundary sphere $\mathbb{S}^{2}=\partial \mathbb{B}_{1}^{3}$ orthogonally, one can choose such an orthonormal basis in the threedimensional space that (i) $\left.N\right|_{p}=(0,0,1)$, (ii) the unit tanget vector to the component of the boundary at $p$ is $(0,1,0)$, (iii) the outward normal vector to $\mathbb{S}^{2}$ at $p$ is $(1,0,0)$. Remark now that if we put the origin at the center of the ball then $p=(1,0,0)$ and the sphere is just the standard unit sphere centered at the origin.

Let us parametrize the surface as $(x, y, f(x, y))$. Then one has

$$
f(1,0)=0, \quad f_{x}(1,0)=f_{y}(1,0)=0 .
$$

Then the component of the boundary can be parametrized as

$$
\omega(t)=(g(t), t, f(g(t), t)) .
$$

Since $\omega^{\prime}(0)=(0,1,0)$ and

$$
\omega^{\prime}(t)=\left(g^{\prime}(t), 1, f_{x}(g(t), t) g^{\prime}(t)+f_{y}(g(t), t)\right),
$$

one has

$$
g(0)=1, \quad g^{\prime}(0)=0 .
$$

Since the surface meets the sphere orthogonally, at each point $\omega(t)$ the unit normal vector

$$
N=\frac{\left(-f_{x},-f_{y}, 1\right)}{\sqrt{1+f_{x}^{2}+f_{y}^{2}}}
$$

is orthogonal to the radius vector of this point, i.e.

$$
-f_{x}(g(t), t) g(t)-f_{y}(g(t), t) t+f(g(t), t)=0 .
$$

If one takes the derivative of equation (9) with respect to $t$ and one substitutes $t=0$, then one obtains $f_{x y}(1,0)=0$ due to formulae (8) .

This implies that

$$
H\left|p=\frac{f_{x x}(1,0)+f_{y y}(1,0)}{2}, \quad K\right| p=f_{x x}(1,0) f_{y y}(1,0) .
$$

Since the surface is minimal, one has $H=0$. It follows that

$$
f_{y y}(1,0)=\sqrt{-K} .
$$


Let us take

$$
l=\int_{0}^{t}\left|\omega^{\prime}(t)\right| d t=\int_{0}^{t} \sqrt{\left(g^{\prime}(t)^{2}+1+\left(f_{x}(g(t), t) g^{\prime}(t)+f_{y}(g(t), t)\right)^{2}\right.} d t
$$

as a natural parameter on the component of the boundary. Then equations (5) and (8) imply

$$
\frac{d l}{d t}(0)=1, \quad \frac{d^{2} l}{d t^{2}}(0)=0 .
$$

It follows that

$$
\frac{d^{2} \omega}{d t^{2}}(0)=\frac{d^{2} \omega}{d l^{2}}(0)\left(\frac{d l}{d t}(0)\right)^{2}+\frac{d \omega}{d l}(0) \frac{d^{2} l}{d t^{2}}(0)=\frac{d^{2} \omega}{d l^{2}}(0) .
$$

Let us take the derivative of equation (7), substitute $t=0$ and use (5) and (8). One obtains

$$
\frac{d^{2} \omega}{d t^{2}}(0)=\left(g^{\prime \prime}(0), 0, f_{y y}(1,0)\right)
$$

Since the boundary belongs to the sphere, equation (5) imples

$$
g(t)^{2}+t^{2}+f(g(t), t)^{2}=1 .
$$

Let us take the second derivative and substitute $t=0$. One obtains

$$
g^{\prime \prime}(0)=-1 \text {. }
$$

Let us now compute the curvature of the boundary at the point $p$. Equations (11), (12), (13) and (10) imply

$$
\left.k\right|_{p}=\left|\frac{d^{2} \omega}{d l^{2}}(0)\right|=\left|\frac{d^{2} \omega}{d t^{2}}(0)\right|=\sqrt{\left(g^{\prime \prime}(0)\right)^{2}+\left(f_{y y}(1,0)\right)^{2}}=\sqrt{1-K} .
$$

Since $K$ is a constant on the connected component of the boundary, the curvature of this component is also a constant. It is well known that a curve of constant curvature on a sphere is an arc of a circle. This finishes the proof.

Proof of Theorem 1.1. Let $A$ be the annulus (1), and $\langle\cdot, \cdot\rangle$ be the standard $\mathbb{C}$-bilinear scalar product on $\mathbb{C}^{3}$.

Consider a map $u: \bar{A} \longrightarrow \overline{\mathbb{B}}_{1}^{3} \subset \mathbb{R}^{3}$ such that $u \in C^{2}(A) \cap C^{1}(\bar{A})$, $u(\partial A) \subset \mathbb{S}^{2}=\partial \mathbb{B}_{1}^{3}$,

$$
u_{z \bar{z}}=0, \quad\left\langle u_{z}, u_{z}\right\rangle=0,
$$

and $u(A)$ meets $\mathbb{S}^{2}$ orthogonally. 
Denote by $N$ the unit normal field on $u(A)$ and by $u_{z z}^{\perp}$ the component of $u_{z z}$ normal to $u(A)$, i.e.

$$
u_{z z}=u_{z z}^{\perp}+\frac{\left\langle u_{z z}, u_{\bar{z}}\right\rangle}{\left\langle u_{z}, u_{\bar{z}}\right\rangle} u_{z}+\frac{\left\langle u_{z z}, u_{z}\right\rangle}{\left\langle u_{z}, u_{\bar{z}}\right\rangle} u_{\bar{z}}
$$

In fact, the second formula from (14) implies that $\left\langle u_{z z}, u_{z}\right\rangle=0$.

Consider polar coordinates $r, \theta$ such that $z=r e^{i \theta}$. Since $u_{r}$ and $u_{\theta}$ are tangent to $u(A)$, one has

$$
u_{z z}^{\perp}=\frac{e^{-2 i \theta}}{4}\left(u_{r r}^{\perp}-\frac{2 i}{r} u_{r \theta}^{\perp}-\frac{1}{r^{2}} u_{\theta \theta}^{\perp}\right) .
$$

The free boundary condition, i.e. the condition that $u(A)$ meets $\mathbb{S}^{2}$ orthogonally, implies $\left.u_{r}\right|_{\partial A} \perp \mathbb{S}^{2}$. Hence, one has $u_{r}=\lambda u$ on $\partial A$ for some function $\lambda$. It follows that

$$
u_{r \theta}=\lambda_{\theta} u+\lambda u_{\theta}=\frac{\lambda_{\theta}}{\lambda} u_{r}+\lambda u_{\theta}
$$

is a tangent vector. This means that $u_{r \theta}^{\perp}=0$ on $\partial A$. Then equation (15) implies that $e^{4 i \theta}\left\langle u_{z z}^{\perp}, u_{z z}^{\perp}\right\rangle$ is real on $\partial A$. Since $z^{4}=r^{4} e^{4 i \theta}$, it follows that $z^{4}\left\langle u_{z z}^{\perp}, u_{z z}^{\perp}\right\rangle$ is real and positive on $\partial A$.

It is well-known that in a simple connected domain for a minimal surface $u$ there exists an adjoint surface $u^{*}$ such that $f=u+i u^{*}$ is holomorphic and $l=\left\langle f^{\prime \prime}, N\right\rangle$ is a holomorphic function (including the branch points), see e.g. [DHS10]. It follows that

$$
z^{4}\left\langle u_{z z}^{\perp}, u_{z z}^{\perp}\right\rangle=\frac{z^{4}}{4} l^{2}
$$

is also a holomorphic function. $A$ is not simply connected, but the property of being holomorphic is local, one can check it in simple connected neighbourhoods of points of $A$. Hence, $z^{4}\left\langle u_{z z}^{\perp}, u_{z z}^{\perp}\right\rangle$ is holomorphic on $A$. Since $z^{4}\left\langle u_{z z}^{\perp}, u_{z z}^{\perp}\right\rangle$ is real on $\partial A$, this function is constant on $A$,

$$
z^{4}\left\langle u_{z z}^{\perp}, u_{z z}^{\perp}\right\rangle=k \in \mathbb{R}, \quad k>0 .
$$

Let us now consider the celebrated Enneper-Weierstrass representation. For $\Phi=2 u_{z}$ there exist a holomorphic function $\mu$ and meromorphic function $\nu \not \equiv 0$ such that $\mu \nu^{2}$ is holomorphic and

$$
\Phi=\left(\frac{1}{2} \mu\left(1-\nu^{2}\right), \frac{i}{2} \mu\left(1+\nu^{2}\right), \mu \nu\right) .
$$

It follows that

$$
u(z)=u_{0}+\operatorname{Re} \int_{z_{0}}^{z} \Phi(z) d z .
$$


Let $z=x+i y$. Let us recall that

$$
\begin{gathered}
d s^{2}=\Lambda\left(d x^{2}+d y^{2}\right), \quad \Lambda=\frac{1}{4}|\mu|^{2}\left(1+|\nu|^{2}\right)^{2}, \\
l=\left\langle f^{\prime \prime}, N\right\rangle=-\mu \nu^{\prime},
\end{gathered}
$$

see e.g. [DHS10]. Remark that $f$ could be multivalued since $A$ is not simply connected, but $f^{\prime}=\Phi$ is a holomorphic function.

Let us recall that $z_{0}$ is a branch point if and only if $z_{0}$ is a zero of $\mu$ and $\mu \nu^{2}$. Since there is no branch points on the boundary, see e.g. [CM11], $\mu$ has no zeroes on $\partial A$.

Let us now consider the point $z_{0}=1$ or $z_{0}=\rho$ on $\partial A$. Remark that any point on $\partial A$ could be transformed by a rotation to one of these two points. Consider the curve $u\left(\left|z_{0}\right| e^{i \theta}\right) \subset \mathbb{S}^{2}$ parametrized by $\theta$.

It is well known that for a curve lying on a sphere its osculating spheres coincide with the initial sphere. It is also well known that the circle obtained as intersection of the osculating sphere and the osculating plane at a point of a curve touches this curve at the second order at this point. It follows that there exist a circle $\gamma(t) \subset \mathbb{S}^{2}$, where $t$ is an affine natural parameter, such that

$$
u\left(z_{0}\right)=\gamma(0), \quad u_{\theta}\left(z_{0}\right)=\dot{\gamma}(0), \quad u_{\theta \theta}\left(z_{0}\right)=\ddot{\gamma}(0) .
$$

Performing, if necessary, a rotation and reflection of $\mathbb{R}^{3}$, we can assume that (i) the circle $\gamma$ is the circle $\gamma^{3}(t)=$ const, (ii) $\gamma^{2}(0)=0$, here the superscripts mean the coordinate number, (iii) $N\left(z_{0}\right) \neq(0,0,1)$. Let us remark that property (i) implies

$$
u_{\theta}^{3}\left(z_{0}\right)=0, \quad u_{\theta \theta}^{3}\left(z_{0}\right)=0,
$$

property (ii) implies

$$
u_{\theta}^{1}\left(z_{0}\right)=0
$$

and (iii) implies that $\nu$ does not have a pole at $z_{0}$, see e.g. [DHS10].

Combining

$$
u_{\theta}=i z u_{z}-i \bar{z} u_{\bar{z}}=-\operatorname{Im}(z \Phi)
$$

with equations (20) and (21), one has

$$
u_{\theta}\left(z_{0}\right)=\left(0,-\operatorname{Re}\left(\left.\frac{z_{0}}{2} \mu\left(1+\nu^{2}\right)\right|_{z=z_{0}}\right), 0\right) .
$$

Computing $\left|u_{\theta}\left(z_{0}\right)\right|$ directly and using formula (18), we obtain the equation

$$
\left|\operatorname{Re}\left(\frac{z_{0}}{2} \mu\left(z_{0}\right)\left(1+\nu^{2}\left(z_{0}\right)\right)\right)\right|=\frac{\left|z_{0}\right|}{2}\left|\mu\left(z_{0}\right)\right|\left(1+\left|\nu\left(z_{0}\right)\right|^{2}\right) \mid
$$


It is easy to prove that for $a, b \in \mathbb{C}$ the inequality

$$
\left|\operatorname{Re}\left(a\left(1+b^{2}\right)\right)\right| \leqslant|a|\left(1+|b|^{2}\right)
$$

holds, and one has the equality if and only if $a, b \in \mathbb{R}$ or $a=0$.

Since $\mu$ has no zeroes on $\partial A$, it follows that $\mu\left(z_{0}\right), \nu\left(z_{0}\right) \in \mathbb{R}$.

Since $\gamma(t)$ is a circle parametrized by an affine natural parameter, $\left(\ddot{\gamma}^{1}(0), \ddot{\gamma}^{2}(0)\right) \|\left(\gamma^{1}(0), \gamma^{2}(0)\right)$. Then $\gamma^{2}(0)=0$ implies

$$
u_{\theta \theta}^{2}\left(z_{0}\right)=\ddot{\gamma}^{2}(0)=0 \text {. }
$$

Since

$$
u_{\theta \theta}=-\operatorname{Re}\left(z \Phi+z^{2} \Phi^{\prime}\right)
$$

one has

$$
u_{\theta \theta}^{2}\left(z_{0}\right)=-\left.\operatorname{Re}\left(z \frac{i}{2} \mu\left(1+\nu^{2}\right)+z^{2} \frac{i}{2}\left(\mu^{\prime}\left(1+\nu^{2}\right)+2 \mu \nu \nu^{\prime}\right)\right)\right|_{z=z_{0}}=0 .
$$

It follows that

$$
\left.\left(\mu^{\prime}\left(1+\nu^{2}\right)+2 \mu \nu \nu^{\prime}\right)\right|_{z=z_{0}} \in \mathbb{R} .
$$

But formula (17) implies $\left.\mu \nu^{\prime}\right|_{z=z_{0}} \in \mathbb{R}$. It follows that $\mu^{\prime}\left(z_{0}\right), \nu^{\prime}\left(z_{0}\right) \in \mathbb{R}$.

Let us remark that

$$
2 \mu\left(z_{0}\right)|\mu|_{\theta}\left(z_{0}\right)=\frac{\partial}{\partial \theta}|\mu|^{2}\left(z_{0}\right)=\left.\left(\mu_{\theta} \bar{\mu}+\mu \bar{\mu}_{\theta}\right)\right|_{z=z_{0}}=\left(2 \operatorname{Re} \mu_{\theta}\left(z_{0}\right)\right) \mu\left(z_{0}\right) .
$$

Since $\mu_{\theta}\left(z_{0}\right)=i z \mu^{\prime}\left(z_{0}\right)$ is purely imaginary and $\mu\left(z_{0}\right) \neq 0$, one has $|\mu|_{\theta}\left(z_{0}\right)=0$. The same argument proves that at least one of quantities $|\nu|_{\theta}\left(z_{0}\right)$ or $\nu\left(z_{0}\right)$ is zero.

Consider now $\sqrt{\Lambda}=\frac{|\mu|}{2}\left(1+|\nu|^{2}\right)$. One has

$$
\frac{\partial}{\partial \theta} \sqrt{\Lambda}\left(z_{0}\right)=\left.\left(\frac{|\mu|_{\theta}}{2}\left(1+|\nu|^{2}\right)+|\mu||\nu||\nu|_{\theta}\right)\right|_{z=z_{0}}=0 .
$$

Since the formula $\Lambda_{\theta}=0$ does not change under rotations of $z$-plane or isometries of $\mathbb{R}^{3}$, it holds for any point on $\partial A$. This means that $\theta$ is an affine natural parameter on each connected component of $u(\partial A)$.

Moreover, the formula for Gaussian curvature, see e.g. [DHS10],

$$
K=-\left(\frac{4\left|\nu^{\prime}\right|}{|\mu|\left(1+|\nu|^{2}\right)^{2}}\right)^{2}
$$

and equations (16), (18) and (19) imply that $K=-\frac{4 k}{|z|^{4} \Lambda^{2}}$ is a constant on each connected component of $u(\partial A)$.

Then Lemma 2.1 implies that each component of $u(\partial A)$ is a circle lying on $\mathbb{S}^{2}$.

Let a circle $\sigma$ be a boundary component of $u(A)$ and $p \in \sigma$. Let us consider the case when $\sigma$ is not a great circle on $\mathbb{S}^{2}$. In this case 
one can find a catenoid $H$ which meets orthogonally $S^{2}$ at $\sigma$. Without loss of generality we can assume that the initial minimal surface could be locally parametrised as $(x, y, f(x, y))$ and the catenoid as $(x, y, h(x, y))$. Since $f$ satisfies the minimal surface equation and have the same Cauchy data on $\sigma$ in a neighbourhood of $p$ as $h$, one has $f(x, y)=h(x, y)$ where both functions are defined. Then $u(A)$ and $H$ coincide globally and $u$ is a reparametrisation of a catenoid. Since all conformal automorphisms of the annulus $A$ are described by Schottky theorem [Sch77,

$$
z \mapsto \lambda z^{ \pm 1}
$$

where $|\lambda|=1$ or $\rho$, we obtain that $u$ is a catenoid.

In the case when $\sigma$ is a great circle the same argument could be applied with a plane disk instead of a cathenoid. As a result, another connected component of $u(\partial A)$ is inside the ball. This contradicts the assumption $u(\partial A) \subset \mathbb{S}^{2}$. This finishes the proof.

Assuming a non-zero Dirichlet boundary condition on $\partial K$ we will consider a generalization of the problem (3):

$$
\begin{cases}\Delta v=0 & \text { in } K \\ v=\alpha|x| & \text { on } \partial K \\ |\nabla v|=1 & \text { on } \partial K \backslash\{0\}\end{cases}
$$

where $v$ is a homogeneous degree 1 function. We assume that $\alpha \in \mathbb{R}$ is a constant. Then the second boundary condition implies that $\alpha \in$ $(-1,1)$.

Let $v$ be a homogeneous order 1 harmonic function defined in the cone $K$ and satisfying equation (23). Denote $G=K \cap \mathbb{S}^{2}, f=\nabla v$ : $G \rightarrow \mathbb{R}^{3}$. Let $\gamma ß G$ be the set of critical points of $f$, i.e., the set of vanishing of the differential $d f$. Since $f$ is a real analytic map, $\gamma$ is either a set of isolated points in $G$, or it contains a one dimensional ark $\gamma^{\prime}$, see the paper [Whi65]. Consider the second case. Let $\widetilde{\gamma}$ be the conic extension of $\gamma^{\prime}$ to $\mathbb{R}^{3}$. Then there is a linear function $l$ in $\mathbb{R}^{3}$ such that $l-v=0, \nabla v-\nabla l=0$ on $\widetilde{\gamma}$. Since $l-v$ is a harmonic function then by the uniqueness of the solution of the Cauchy problem $l=v$ in $K$ and the theorem follows. Suppose now that $\gamma^{\prime}$ is a set of isolated points. By Proposition 1.3 surface $\nabla v\left(G \backslash \gamma^{\prime}\right) B \mathbb{R}^{3}$ is a minimal surface. By a theorem by Gulliver and Lawson, see [GL86] and [Mee07], the surface $\Gamma$ could be extended to the set $\gamma^{\prime}$ as a branching minimal surface $\Gamma=\nabla v(G)$. Assume now that $v$ satisfies in $K$ equation (3). Since $|\nabla v|=1, \partial \Gamma ß \mathbb{S}^{2}$. Let $x \in \partial K$. Since $v$ is a homogeneous order 1 function $\nabla v(x)=\alpha x+\beta e$, where $|e|=1,(x, e)=0, \sqrt{\alpha^{2}+\beta^{2}}=1$. Thus the angle between vectors $x$ and $\nabla v(x)$ is fixed for all points 
$x \in \partial K$. By Proposition $1.3 x$ is a unit normal to $\Gamma$ at $\nabla v(x)$ and hence the angle between vectors $N(\nabla v(x))$ and $\nabla v(x)$ is fixed for all points $x \in \partial K$. Thus $\Gamma$ intersects sphere $\mathbb{S}^{2}$ under a fixed angle in particular if $\alpha=0$ then $\Gamma$ meats sphere $\mathbb{S}^{2}$ orthogonally. Now Theorem 1.2 follow from the theorem of Nitsche and from Theorem 1.1.

Remark. It is easy to see that the curves $\partial \Gamma$ and $\partial K \cap \mathbb{S}_{r}^{2}$ are dual curves on the sphere $\mathbb{S}_{r}^{2}$.

Proof of Theorem 1.5. Since the Gaussian curvature of $\Omega$ is 1 there exists an isometry

$$
i: \Omega \longrightarrow \mathbb{S}^{2}
$$

and $i(\Omega)$ is a domain possibly multi-sheeted on $\mathbb{S}^{2}$. Denote by $u$ the pull down of the function $v$ from $\Omega$ to $i(\Omega) B \mathbb{S}^{2}$. We will assume that the function $u$ is extended as a homogeneous 1 function to a cone $K \beta \mathbb{R}^{3}$ over $i(\Omega)$, where $K$ is possibly multi-sheeted cone. Then $u$ satisfies equation (23). The same argument as above shows that the surface $\nabla u$ intersects sphere $\mathbb{S}_{r}^{2}$ under a fixed angle. Hence Theorem 1.5 follows from Nitsche's theorem.

\section{REFERENCES}

[AC81] H. W. Alt and L. A. Caffarelli, Existence and regularity for a minimum problem with free boundary, J. Reine Angew. Math. 325 (1981), 105-144. MR 618549

[BCN97] Henri Berestycki, Luis Caffarelli, and Louis Nirenberg, Further qualitative properties for elliptic equations in unbounded domains, Ann. Scuola Norm. Sup. Pisa Cl. Sci. (4) 25 (1997), no. 1-2, 69-94 (1998), Dedicated to Ennio De Giorgi. MR 1655510

[Bla21] Wilhelm Blaschke, Vorlesungen über Differentialgeometrie und geometrische Grundlagen von Einsteins Relativitätstheorie. Band I. Elementare Differentialgeometrie, Springer, Berlin, 1921.

[BZ80] Carlos A. Berenstein and Lawrence Zalcman, Pompeiu's problem on symmetric spaces, Comment. Math. Helv. 55 (1980), no. 4, 593-621. MR 604716

[CJK04] Luis A. Caffarelli, David Jerison, and Carlos E. Kenig, Global energy minimizers for free boundary problems and full regularity in three dimensions, Noncompact problems at the intersection of geometry, analysis, and topology, Contemp. Math., vol. 350, Amer. Math. Soc., Providence, RI, 2004, pp. 83-97. MR 2082392

[CM11] Tobias Holck Colding and William P. Minicozzi, II, A course in minimal surfaces, Graduate Studies in Mathematics, vol. 121, American Mathematical Society, Providence, RI, 2011. MR 2780140

[CS05] Luis Caffarelli and Sandro Salsa, A geometric approach to free boundary problems, Graduate Studies in Mathematics, vol. 68, American Mathematical Society, Providence, RI, 2005. MR 2145284

[DHS10] Ulrich Dierkes, Stefan Hildebrandt, and Friedrich Sauvigny, Minimal surfaces, second ed., Grundlehren der Mathematischen Wissenschaften 
[Fundamental Principles of Mathematical Sciences], vol. 339, Springer, Heidelberg, 2010, With assistance and contributions by A. Küster and R. Jakob. MR 2566897

[DHT10a] Ulrich Dierkes, Stefan Hildebrandt, and Anthony J. Tromba, Global analysis of minimal surfaces, second ed., Grundlehren der Mathematischen Wissenschaften [Fundamental Principles of Mathematical Sciences], vol. 341, Springer, Heidelberg, 2010. MR 2778928

[DHT10b] — Regularity of minimal surfaces, second ed., Grundlehren der Mathematischen Wissenschaften [Fundamental Principles of Mathematical Sciences], vol. 340, Springer, Heidelberg, 2010, With assistance and contributions by A. Küster. MR 2760441

[DSJ09] Daniela De Silva and David Jerison, A singular energy minimizing free boundary, J. Reine Angew. Math. 635 (2009), 1-21. MR 2572253

[FL14] Ailana Fraser and Martin Man-chun Li, Compactness of the space of embedded minimal surfaces with free boundary in three-manifolds with nonnegative Ricci curvature and convex boundary, J. Differential Geom. 96 (2014), no. 2, 183-200. MR 3178438

[FPZ17] Abigail Folha, Frank Pacard, and Tatiana Zolotareva, Free boundary minimal surfaces in the unit 3-ball, Manuscripta Math. 154 (2017), no. 3-4, 359-409. MR 3713919

[FS11] Ailana Fraser and Richard Schoen, The first Steklov eigenvalue, conformal geometry, and minimal surfaces, Adv. Math. 226 (2011), no. 5, 4011-4030. MR 2770439

[FS15] _ Uniqueness theorems for free boundary minimal disks in space forms, Int. Math. Res. Not. IMRN (2015), no. 17, 8268-8274. MR 3404014

[FS16] Sharp eigenvalue bounds and minimal surfaces in the ball, Invent. Math. 203 (2016), no. 3, 823-890. MR 3461367

[GL86] Robert Gulliver and H. Blaine Lawson, Jr., The structure of stable minimal hypersurfaces near a singularity, Geometric measure theory and the calculus of variations (Arcata, Calif., 1984), Proc. Sympos. Pure Math., vol. 44, Amer. Math. Soc., Providence, RI, 1986, pp. 213-237. MR 840275

[HL82] Reese Harvey and H. Blaine Lawson, Jr., Calibrated geometries, Acta Math. 148 (1982), 47-157. MR 666108

[JS15] David Jerison and Ovidiu Savin, Some remarks on stability of cones for the one-phase free boundary problem, Geom. Funct. Anal. 25 (2015), no. 4, 1240-1257. MR 3385632

[Lew68] Hans Lewy, On the non-vanishing of the jacobian of a homeomorphism by harmonic gradients, Ann. of Math. (2) 88 (1968), 518-529. MR 0232007

[LLR88] Rémi Langevin, Gilbert Levitt, and Harold Rosenberg, Hérissons et multihérissons (enveloppes parametrées par leur application de Gauss), Singularities (Warsaw, 1985), Banach Center Publ., vol. 20, PWN, Warsaw, 1988, pp. 245-253. MR 1101843

[Mee07] William H. Meeks, III, The structure of stable minimal surfaces near a singularity, Michigan Math. J. 55 (2007), no. 1, 155-161. MR 2320177 
[Nit72] Johannes C. C. Nitsche, On the boundary regularity of surfaces of least area in Euclidean space, Continuum mechanics and related problems of analysis (on the occasion of the eightieth birthday of Academician N. I. Mushelišvili) (Russian), Izdat. "Nauka", Moscow, 1972, pp. 371-373. MR 0394393

[NS15] Nikolai Nadirashvili and Yannick Sire, Maximization of higher order eigenvalues and applications, Mosc. Math. J. 15 (2015), no. 4, 767-775. MR 3438833

[RRS17] Antonio Ros, David Ruiz, and Pieralberto Sicbaldi, A rigidity result for overdetermined elliptic problems in the plane, Comm. Pure Appl. Math. 70 (2017), no. 7, 1223-1252. MR 3666566

[RS97] Antonio Ros and Rabah Souam, On stability of capillary surfaces in a ball, Pacific J. Math. 178 (1997), no. 2, 345-361. MR 1447419

[Sch77] Friedrich Hermann Schottky, Über konforme Abbildung von mehrfach zusammenhängenden Fläche, J. Reine Angew. Math. 83 (1877), 300351.

[Sch01] Philip W. Schaefer, On nonstandard overdetermined boundary value problems, Proceedings of the Third World Congress of Nonlinear Analysts, Part 4 (Catania, 2000), Nonlinear Anal. 47 (2001), no. 4, pp. 22032212. MR 1971630

[Tra14] Martin Traizet, Classification of the solutions to an overdetermined elliptic problem in the plane, Geom. Funct. Anal. 24 (2014), no. 2, 690-720. MR 3192039

[WCG95] N. B. Willms, Marc Chamberland, and G. M. L. Gladwell, A duality theorem for an overdetermined eigenvalue problem, Z. Angew. Math. Phys. 46 (1995), no. 4, 623-629. MR 1345815

[Whi65] Hassler Whitney, Local properties of analytic varieties, Differential and Combinatorial Topology (A Symposium in Honor of Marston Morse), Princeton Univ. Press, Princeton, N. J., 1965, pp. 205-244. MR 0188486

[Zal80] Lawrence Zalcman, Offbeat integral geometry, Amer. Math. Monthly 87 (1980), no. 3, 161-175. MR 562919

(Nikolai S. Nadirashvili) CNRS, I2M UMR 7353 - Centre de MathÉmatiques et Informatique, Marseille, France

E-mail address: nnicolas@yandex.ru

(Alexei V. Penskoi) Department of Higher Geometry and Topology, Faculty of Mathematics and Mechanics, Moscow State University, Leninskie Gory, GSP-1, 119991, Moscow, Russia

and

Faculty of Mathematics, National Research University Higher School of Economics, 6 Usacheva Str., 119048, Moscow, Russia

and

Interdisciplinary Scientific Center J.-V. Poncelet (ISCP, UMi 2615), Bolshoy Vlasyevskiy Pereulok 11, 119002, Moscow, Russia

E-mail address, Corresponding author: penskoi@mccme.ru 